\title{
Frédéric VIGUIER, La Cause des pauvres en France
}

\section{Marc-Antoine Sabaté}

\section{OpenEdition}

\section{Journals}

Édition électronique

URL : https://journals.openedition.org/ress/7316

DOI : $10.4000 /$ ress. 7316

ISBN : $1663-4446$

ISSN : 1663-4446

Éditeur

Librairie Droz

\section{Édition imprimée}

Date de publication : 6 décembre 2021

Pagination : 317-321

ISSN : 0048-8046

Référence électronique

Marc-Antoine Sabaté, "Frédéric VIGUIER, La Cause des pauvres en France », Revue européenne des sciences sociales [En ligne], 59-2 | 2021, mis en ligne le 01 décembre 2021, consulté le 09 décembre 2021. URL : http://journals.openedition.org/ress/7316 ; DOI : https://doi.org/10.4000/ress.7316

Ce document a été généré automatiquement le 9 décembre 2021.

(C) Librairie Droz 


\title{
Frédéric VIGUIER, La Cause des pauvres en France
}

\author{
Marc-Antoine Sabaté
}

\section{RÉFÉRENCE}

Frédéric VIGUIER, 2020, La Cause des pauvres en France, Paris, Presses de Sciences Po, $362 \mathrm{p}$.

1 Au mois de janvier 1995, quatorze ans après avoir fameusement déclaré la « crise de l'État-providence ", Pierre Rosanvallon prolongeait son diagnostic par un essai annonçant l'avènement d'une Nouvelle question sociale fondée sur « l'exclusion » plutôt que sur «l'exploitation". L'ouvrage avait fait date et demeure aujourd'hui une référence obligée. Il faut dire que quelques années après la mise en place du Revenu minimum d'insertion (RMI) en 1988, et à la veille d'une campagne présidentielle remportée cette année-là par un Jacques Chirac surfant au printemps sur le thème de la « fracture sociale » avant de se heurter, l'automne venu, à un mouvement social inégalé depuis les grèves de mai 1968, le propos de Rosanvallon fixait un certain air du temps. Celui, bien-sûr, d'une remise en cause croissante de l'État social, dont les institutions seraient désormais "inadaptée[s] aux transformations de l'économie » (p.327), mais aussi et peut-être surtout celui de l'accession de la pauvreté au statut de "problème public » (p.19) et de la formation d'un "consensus » politique "sur la nécessité de protéger les pauvres et les exclus» (p.6). Tel est précisément l'objet de l'ouvrage de Frédéric Viguier, La Cause des pauvres en France, qui s'intéresse à la genèse de cet air du temps et interroge l'écart entre le triomphe de la "compassion » au tournant des années 1980-1990 et son « usure » (p. 6) depuis le début des années 2000.

Car le fait est là, constate Viguier : « le discours sur la pauvreté s'est durci » ces deux dernières décennies. À « l'ardente nécessité de la lutte contre l'exclusion ont succédé les mises en gardes contre les fraudeurs supposés, le désir d'intensifier le contrôle des bénéficiaires du revenu minimum et des allocations-chômage, et les exhortations à se 
lever de bonne heure ou à traverser la rue pour trouver du travail» (p. 5). Comment en est-on arrivé là ? - se demande-t-il alors, inscrivant sa réflexion dans le sillage de la sociohistoire définie par Gérard Noiriel comme une " histoire problème ", cherchant à faire ressortir la manière dont «le passé pèse inconsciemment sur nos représentations » (p. 9). Là, c'est-à-dire à l'hégémonie de «la norme d'autonomie des assistés, cette idée que les pauvres doivent se mobiliser activement pour sortir de leur condition ». Pour Viguier, « le basculement ne date pas des années 2000 mais remonte plus loin, à la mise en place de formes individualisées de traitement de la pauvreté et du chômage dès les années 1980 » (p. 6-8). Formes nouvelles dont l'essai de Rosanvallon venait précisément donner le principe : appréhension de la pauvreté et de l'exclusion non pas comme des risques sociaux mais comme autant de trajectoires individuelles devant être traitées de manière singulière, par un « réformisme de l'individu » fondé sur une logique contractuelle d'activation.

On ne saurait toutefois s'arrêter là, explique Viguier. Les acteurs visibles et audibles de la lutte contre la pauvreté n'ont en effet pas attendu les années 1980 pour faire valoir leur cause. Contrairement au sens commun, partagé tant par le récit rosanvallonien de la crise de l'État-providence que par les critiques du tournant de l'activation et de la conditionnalisation de la protection sociale, «l'invention de techniques réparatrices des sujets [...] telle que l'insertion ou l'accompagnement individualisé » (p.337) remonte en réalité bien avant le tournant des années 1980-1990 qui, en tant quel tel, marque donc plutôt l'achèvement d'un long processus d'institutionnalisation de la pauvreté conçue comme un problème spécifique, appelant «des moyens d'action spécifiques » (p. 12). C'est en fait dès les années 1950 - avec pour "mythe fondateur » l'appel de l'abbé Pierre de l'hiver 1954 (p. 74) - qu'apparait ce que Viguier propose de regarder comme une « cause », c'est-à-dire la "volonté proclamée par divers acteurs et groupes mobilisés de réparer l'injustice faite à un groupe social en portant à la reconnaissance publique son existence jusqu'alors ignorée ou sous-estimée » (p. 8).

L'enquête s'intéresse alors aux acteurs de cette cause des pauvres, à commencer par ses avocats originels: les associations caritatives «issues de la gauche du catholicisme social» (p. 67) qui, en marge à la fois du mouvement ouvrier et des grandes œuvres religieuses, ont inventé et mis en œuvre un «nouveau rapport aux pauvres et à la pauvreté » (p. 8) ; rapport fondé sur l'organisation d'une " autopromotion des pauvres par eux-mêmes" (p.120), en rupture avec la charité traditionnelle accusée de maintenir « les pauvres dans la dépendance au lieu de leur permettre de s'insérer dans la société" (p. 109). C'est principalement sur le cas d'ATD Quart Monde que se concentre l'étude : sa création à la fin des années 1950 par le père Joseph Wresinski et son investissement auprès du "peuple des pauvres" dans les bidonvilles; son invention du "volontariat", à mi-chemin entre bénévolat et travail social, comme "apostolat moderne et laïque» (p. 79); son développement d'une "lecture comportementaliste de la pauvreté ", influencée par la "science de la pauvreté » américaine et les "programmes d'action sociale communautaire dits d'empowerment» (p 91); sa mobilisation politique, enfin, donnant à entendre la parole des pauvres et plaidant pour la mise en place d'un programme d'aide et d'action sociale comprenant notamment l'introduction d'un «revenu minimum garanti» (p. 229). Viguier montre ainsi très bien comment ATD Quart Monde a participé à l'autonomisation de la question de la pauvreté et à l'émergence d'un nouveau 
référentiel d'action publique se donnant pour objectif de «transformer les exclus en individus autonomes» (p. 309).

Mais c'est également ce qu'il nomme «l'espace de la cause des pauvres » que Viguier étudie. Un espace certes d'abord délimité par les associations de lutte contre la pauvreté, mais au sein duquel, au fur et à mesure de son "élargissement » et de ses «reconfigurations» (p.8), se sont également inscrits travailleurs sociaux, hauts fonctionnaires, intellectuels et représentants politiques. À cet égard, l'ouvrage présente deux qualités qui méritent d'être soulignées.

D'une part, le sociologue découvre et démontre, au fil de son enquête, le rôle du catholicisme social comme facteur déterminant de l'avancée de la cause des pauvres. Matrice qui ne joue pas tant à un niveau directement religieux qu'au niveau de la socialisation des acteurs. Que l'on s'intéresse aux volontaires associatifs ou aux fonctionnaires réformateurs, on retrouve en effet des trajectoires personnelles marquées, par exemple, par un engagement de jeunesse dans le mouvement jociste, et héritant d'un "antilibéralisme catholique» (p.78) critique de "la violence du capitalisme qui détruit des communautés humaines » (p. 267) et vecteur naturel d'une attention " aux souffrances sociales les plus intolérables » (p. 342). Catholicisme social comme «ethos partagé » (p. 167) qui permet notamment de comprendre le relais que trouveront les associations auprès d'une "deuxième gauche » (p. 342) qui jouera un rôle clef dans l'introduction du RMI, et dont les héritiers se retrouvent aujourd'hui autour du revenu de base universel et inconditionnel.

D'autre part, l'une des principales forces de l'enquête réside dans le geste - où l'on devine l'héritage d'une sociologie de l'action publique soucieuse, après Theda Skocpol, de "ramener l'État au cœur du débat " - consistant à ne pas se contenter de penser l'espace de la cause des pauvres comme se déployant simplement "contre l'État " (p. 174). Viguier décrit en effet avec minutie à la fois l'investissement des différentes sphères de l'État par les associations et la manière dont la cause des pauvres fut relayée et reconfigurée par des « luttes dans l'État» (p. 338). Outre les figures prophétiques et leurs disciples, une attention toute particulière est ainsi réservée aux figures administratives de la cause des pauvres. À René Lenoir notamment: théoricien précurseur de l'exclusion et "figure emblématique » d'une "nouvelle génération de hauts fonctionnaires du social ", réinvestissant dans l'aide et l'action sociale leur énergie réformiste frustrée, dès la fin des années 1960, par le verrouillage gestionnaire de la Sécurité sociale et l'affaiblissement d'un « ethos » administratif un temps incarné par Pierre Laroque. Le passage à la postérité du «lenoirisme "symbolisant alors le «basculement des efforts réformistes depuis la régulation du salariat vers l'amélioration de l'assistance»(p.156-157). D'une actualité plus récente, Viguier s'intéresse également à la figure de Martin Hirsch, à propos duquel il souligne notamment le renversement symbolique qu'il incarne : c'est en effet la "légitimité acquise dans le bénévolat associatif », en tant que président d'Emmaüs, qui permit à ce conseiller d'État, "techno» engagé auprès des "plus défavorisés » (p. 288-290), d'accéder au gouvernement pour y conduire la réforme du Revenu de solidarité active (RSA).

8 Reste à évoquer la question la plus déterminante, celle qui traverse l'ensemble de l'ouvrage : l'autonomisation progressive de la cause des pauvres par rapport à la cause salariale. Le propos de Viguier est, à cet égard encore, à la fois méticuleux et éclairant. Il rappelle tout d'abord comment, pour les créateurs de la Sécurité sociale et pour le 
mouvement ouvrier, Parti Communiste en tête, la cause des pauvres était "dominée " après-guerre; pourquoi elle ne se distinguait pas de "celle de tous les travailleurs exploités» (p. 21-23). Il insiste également, pour en finir avec l'illusion du «mythe glorieux » de 1945, sur le "caractère incomplet de l'œuvre sociale de la Libération » (p. 339). Inachèvement qui explique qu'avant même l'irruption de la « crise économique du milieu des années 1970 » (p. 143) et la médiatisation de la "nouvelle pauvreté » (p. 198-199), associations caritatives, intellectuels et hauts fonctionnaires aient pris le parti des «laissés pour compte de la croissance»(p.95). À cet égard, le principal enseignement de la généalogie de l'exclusion proposée ici est que non seulement le référentiel d'action publique qui s'imposera dans les années 1980, mais également les conditions du doute quant à « la capacité de l'extension du salariat à répondre » à la persistance de la pauvreté (p. 89), étaient déjà pleinement établis au tournant des années 1970.

Or c'est bien cette définition précoce des moyens et des fins de la lutte contre la pauvreté et l'exclusion qui, pour le sociologue, permet de ressaisir la situation actuelle dans son épaisseur historique. Le "travail de construction symbolique des pauvres" mené par les associations a fondamentalement été un « combat pour la reconnaissance de la réalité sous-prolétarienne »; il s'agissait de "faire reconnaître l'existence d'une strate historiquement exclue du monde populaire»(p. 265). Et cette lutte pour la reconnaissance s'est par conséquent déployée en rupture, voire même en opposition franche au mouvement ouvrier et à l'horizon de l'intégration de la classe ouvrière au sein de ce que Robert Castel avait nommé la «société salariale ». Ainsi, dès la fin des années 1960, les «horizons [du] combat» d'ATD Quart Monde étaient clairement annoncés: "amplifier et réformer l'action sociale plutôt qu'inscrire les "sousprolétaires" dans le mouvement de salarisation des classes populaires ou bien dans les circuits ordinaires de la protection sociale assurantielle» (p. 135). Mais ce qui avait été pensé comme une lutte minoritaire a, pour parler comme Castel, progressivement fait retour depuis les marges vers le centre. L'exclusion, explique Viguier, est désormais "pensée comme la menace ordinaire qui pèse sur une majorité des classes populaires ». Il ne s'agit pas là pour le sociologue de mettre en accusation les acteurs de la lutte contre la pauvreté : on ne peut tout d'abord pas éluder « la réalité tangible du déclin de la norme du travail salarié à plein temps ", et les associations caritatives n'ont eu de cesse, rappelle-t-il, de s'opposer au « tournant rigoriste » de la protection sociale et à la responsabilisation/stigmatisation des chômeurs et des pauvres (p.311-313). Il n'empêche, la cause des pauvres a selon lui fini par « échapper à ses avocats » (p. 9). Et c'est bien l'extension des méthodes d'aide et d'action sociale - leur transformation en une " protection minimale généralisée pour le monde des classes populaires peu, mal ou pas salariées » (p. 338) - qui a abouti non pas à intégrer le sous-prolétariat dans le noyau stable du salariat mais, au contraire, à institutionnaliser un « travailleur précaire assisté » (p. 291), créant ainsi ce que Nicolas Duvoux a nommé un "continuum de vulnérabilité » entre les pauvres et les classes populaires.

C'est pourquoi ce qui intéresse Viguier est en définitive la représentation des classes populaires : « déclin d'une identité collective [...] basée sur le travail », « démobilisation politique", mais aussi avènement d'une "vision des classes populaires comme partiellement responsables de la domination qu'elles subissent» (p.11). Le sociologue ne fait ici pas mystère de sa conviction : la "représentation de la pauvreté » qui a émergé dans l'espace de la cause des pauvres a pour principal défaut de l'avoir isolée « du système global des inégalités qui l'engendre» (p.12), quand «seule une action 
centrale sur les inégalités peut, in fine, résorber la pauvreté des exclus» (p. 196). Ce n'est dès lors, selon lui, « pas tant la thèse de l'exclusion qui pose problème que le fait qu'elle entre en concurrence avec celle de l'exploitation au lieu de se combiner avec elle»(p.13). Toujours est-il que, face à la question de la reconnaissance du sousprolétariat et de son articulation aux classes populaires dans les luttes sociales, l'auteur reste finalement, sinon indécis, du moins prudent. En effet, si certains n'ont pas hésité à voir dans les mobilisations du Lumpenproletariat une raison directe de la défaite historique de la cause de l'égalité, Viguier considère quant à lui que si « la cause des pauvres ne peut guère avoir de succès durable si c'est aux dépens de celle des salariés ", « inversement, la cause des salariés souffre d'ignorer la cause des pauvres» (p.13). Reste dès lors à tirer toutes les leçons de l'esseulement des associations «dans leur dénonciation des ravages du capitalisme dérégulé maintenant qu'est profondément affaibli le mouvement ouvrier dont elles déploraient autrefois l'oubli des pauvres » (p. 336). Nul doute que l'ouvrage de Viguier, s'il n'y apporte donc pas de réponse définitive, fournit en revanche les ressources nécessaires pour y faire face.

\section{AUTEURS}

\section{MARC-ANTOINE SABATÉ}

Wiener-Anspach Foundation, University of Cambridge 